Not to appear in Nonlearned J., 45.

\title{
Evidence of $V$-band polarimetric separation of carbon stars at high Galactic latitude
}

\author{
Aruna Goswami, Sreeja S. Kartha \\ Indian Institute of Astrophysics, Bangalore 560034, India \\ e-mail: aruna@iiap.res.in \\ and \\ Asoke K. Sen \\ Department of Physics, Assam University, Silchar, Assam, India
}

\begin{abstract}
Polarization is an important indicator of stellar evolution, especially for stars evolving from red-giant stage to planetary nebulae. However, not much is known about the polarimetric properties of the carbon-enhanced metal-poor (CEMP) stars, although they have been well studied in terms of photometric as well as lowand high-resolution spectroscopy. We report here first-ever estimates of $V$-band polarimetry of a group of CEMP stars. $V$-band polarimetry was planned as the $V$-band is known to show maximum polarization among BVRI polarimetry for any scattering of light caused due to dust. Based on these estimates the program stars show a distinct classification into two: one with $p \%<0.4$ and the other with $p \%>1$. Stars with circumstellar material exhibit a certain amount of polarization that may be caused by scattering of starlight due to circumstellar dust distribution into non-spherically symmetric envelopes. The degree of polarization increases with asymmetries present in the geometry of the circumstellar dust distribution. Our results reflect upon these properties. While the sample size is relatively small, the polarimetric separation of the two groups $(p \%<0.4$ and $p \%$ $>1$ ) is very distinct; this finding, therefore, opens up an avenue of exploration with regard to CEMP stars.
\end{abstract}

Subject headings: stars: carbon stars - stars: chemically peculiar - stars: latetype - stars: low-mass 


\section{Introduction}

A characteristic property of stars evolving from red giant stage to planetary nebulae $(\mathrm{PNe})$ is polarization; observed polarization reaches a maximum during the proto-planetary stage and gradually decreases as the star evolves into a PN (Johnson \& Jones 1991). Polarimetric studies of post-asymptotic giant branch (AGB) stars by Trammell et al. (1994) and Parthasarathy \& Jain (1993) have helped to identify aspherical morphology shells that originate during mass-loss phase as common features of these objects. Quasi-periodic variations in linear polarization are known to be observed in many late-type variables. However, not much work has been done on polarization of carbon stars; we report here $V$-band polarimetric measurements of a sample of carbon stars at high Galactic latitude. Polarization of carbon stars are known to show a flatter wavelength dependence than the oxygen-rich stars. Stars with circumstellar materials exhibit a certain amount of linear polarization. The degree of polarization increases with asymmetries present in the geometry of the shells. By modeling the observed polarization, it is possible to study the geometry and structure of the shells. Polarization models of circumstellar grain scattering shows that graphites do not adequately explain the mean polarimetric behavior of carbon stars (Raveendran 1991). Rowan-Robinson \& Harris (1983) have shown that graphites also do not adequately explain the IR spectra of circumstellar dust envelopes around carbon-rich objects. It was suggested by Czyzak et al. (1982) that graphite formation in circumstellar envelopes is not a very likely process and its likely that carbon grains exist most likely as amorphous carbon.

While Parthasarathy et al. (2005) find no significant variation in polarization for HD 100746 at U, B, I bands, etc., Raveendran (1991) finds mild wavelength dependence of polarization in the case of a few carbon stars of Mira variables. In particular, the polarization was found to increase systematically toward the red in RT Pup, UU Aur shows a dip in the polarization curve around the $V$ band, and $\mathrm{X}$ Vel shows a larger polarization in the $R$ band than in the $V$ band. Multiband polarimetric observations by Serkowski et al. (1975) showed that the interstellar polarization has a peak occurring at a median wavelength $\lambda=0.545 \mu \mathrm{m}$. The maximum effect of interstellar polarization can be felt, therefore, in the yellow spectral region. It is important therefore to undertake follow-up observations on several occasions to study the variations in polarization particularly for those that show significant polarization in the $V$ band. Our present sample includes faint high latitude carbon stars from Christlieb et al. (2001). We have studied a large fraction of this sample based on low- as well as highresolution spectroscopy (Goswami (2005); Goswami et al. (2006, 2007, 2010b); Goswami \& Aoki (2010a)). A primary characteristics of these stars is that they provide evidence of nucleosynthesis of the low- and intermediate-mass stars as most of them are polluted by now-extinct AGB stars. The basic parameters of the program stars are listed in Table 1. 


\section{Observations and data reduction}

Observations were made using $2 \mathrm{~m}$ telescope of IUCAA, Pune using the polarimeter (IMPOL) attached to it. The polarimeter available at the cassegrain focus of $2 \mathrm{~m}$ IUCAA telescope has a rotating half-wave plate (HWP) and Wollaston prism through which light passes before forming a pair of images of an object on the CCD (Ramprakash et al. 1998). The HWP can rotate in several discrete steps, such that its fast axis makes angles $(\alpha)$ with some reference direction (generally celestial north-south). The light which is transmitted out of the Wollaston prism forms two images of any celestial source on the CCD, with the ordinary and extraordinary set of rays. For each object observations were taken at four different positions of the HWP: $0^{0}, 22^{0} .5,45^{0}$ and $67^{0} .5$.

Among BVRI polarimetry, the $V$ band is known to show the maximum polarization (Serkowski et al. 1975) and is ideal for the detection of polarization caused due to scattering by dust. Moreover, because of a low photon flux in the $B$ band, the polarization measurements in the $B$ band often have large errors. For these reasons, we have conducted only $V$-band polarimetry for the program stars. In addition to the program stars we have observed three polarization standard stars and one unpolarized standard star for polarimetric calibration.

Data reduction was carried out using various tasks in IRAF. The task PHOT in APPHOT was used to measure the stellar flux. Zero polarization standard stars were observed to check for any possible instrumental error which proved to be smaller than $0.1 \%$. For stars which show low degree of polarization, measurement accuracy is an important issue. The measured errors are smaller than $0.1 \%$ for majority of the observed stars.

\section{Results and Discussions}

$V$-band polarimetric measurements for the standard polarized and unpolarized stars are presented in Table 2. Estimates from literature are also listed for a comparison. A close agreement between the estimated and literature values lends support to the reliability of our results. The estimated error in polarization is less than or near about $0.1 \%$ for all stars (Table 3) except for the star HD 209621 for which the error is \pm 0.23 . Since polarimetric error in our case is photon noise dominated, in the case of very bright stars this error becomes close to zero. Our program stars include objects brighter as well as fainter than HD 209621. Thus, the error in HD 209621 can be considered to be a typical error value.

The polarization standard stars HD 147084 and HD 160529, both observed on the same

night, show an offset of about $\sim-10^{0}$; the near equality of the offsets is as expected for 
consistency, and a similar offset was also reported earlier by Sen et al. (2000). The offset is essentially a result of constraints in instrumental mounting, such as constraint in mounting the polarimeter with the axis of fixed HWP making as angle $0^{0}$ with celestial north-south axis (this will give 0 offset). We have applied an offset of $-10^{0}$ to the estimated position angles of all the stars observed on the same night as that for HD 147084 and HD 160529. The

expected final values of position angles $\left(\theta^{0}\right)$, obtained with the necessary offset corrections are listed in Table 3.

The linear polarization $(p \%)$, with the errors in the measurements of the program stars, is presented (Table 3) without any corrections applied to the observed values for interstellar polarization. For stars with zero polarization $(\leq 0.1 \%)$ the position angles are not defined, hence position angles are listed only for stars with non-zero polarization. The interstellar polarization is presumed to be negligible because of their high Galactic latitudes. The interstellar reddening, listed in Table 3, for the program stars is small. For the program stars for which color excess have been known, it is instructive to compare the spatial distribution of the color excess with polarization. In Figure 1 (upper panel), we have plotted the $V$-band polarimetric estimates of the stars $(p \%)$ against interstellar reddening $E(B-V)$ along with the mean interstellar polarization $\sim 3 E(B-V)$; (Parthasarathy et al. 2005). Estimated $V$-band polarization is found to be larger than the mean interstellar contribution to the polarization in most of the cases indicating that they are intrinsically polarized.

Polarization does not seem to have any co-relation w.r.t. metallicity $([\mathrm{Fe} / \mathrm{H}])$ of the stars (Figure 1, middle panel). The observed $V$-band percentage polarizations are larger for high Galactic latitude objects (Figure 1, lower panel); however there are two outliers (shown with triangles in Figure 1, lower panel) that are high Galactic latitude objects but show weaker polarization. These two objects (HD 100764 and HE 1152-0355 with $[\mathrm{Fe} / \mathrm{H}] \sim-0.6$ and -1.3 , respectively) are not so metal-poor compared to the other stars that show larger polarization. It has been observed that the objects clearly fall into two distinct groups; five objects showing polarization below $0.4 \%$ and the other five showing polarization above 1.0\%. HD 206983 and HD 196944 in our sample do not show significant evidence of linear polarization in the $V$ band. The properties of the individual stars are discussed below.

\subsection{HD 206983, HD 196944}

HD 206983 is listed in the CH star catalog of Bartkevicius (1996). Recent high-resolution analysis of HD 206983 by Drake \& Pereira (2008) shows this object to be a Barium star with an effective temperature of $4200 \mathrm{~K}$ and a metallicity of $[\mathrm{Fe} / \mathrm{H}]=-1.43$. Estimated $p \%$ is $0.06 \pm 0.13$. The $s$-process element-rich star HD 196944 is one of the first stars recorded to 
have shown very high abundance of lead $(\mathrm{Pb})$. This star, also known as a lead star, is hotter $(\sim 5200 \mathrm{~K})$ and more metal-poor $([\mathrm{Fe} / \mathrm{H}]=-2.25)$ than HD 206983 Aoki et al. (2002); Van Eck et al. (2001)). Both $\mathrm{C}$ and $\mathrm{N}$ abundances are enhanced $([\mathrm{C} / \mathrm{Fe}]=1.2,[\mathrm{~N} / \mathrm{Fe}]=$ 1.3). This is a high-velocity object with estimated radial velocity $-174.76 \pm 0.36 \mathrm{~km} \mathrm{~s}^{-1}$, the abundance distribution observed in this star is interpreted as resulting from now-extinct companion AGB star. HD 196944 is mentioned in SIMBAD as a star with envelop of CH; the envelop might have resulted from as the companion star evolved through AGB and PN phases suffering mass loss. The estimated polarization $(p \%=0.09 \pm 0.02)$ is not significant; it is also likely that the non-detection of polarization would perhaps mean that the $\mathrm{CH}$ envelop is symmetrically structured. No previous polarization estimates are available for these two objects.

\subsection{HD 100764, HD 168986, HD 198269, HD 209621, HE 1152-0355}

Estimated $p \%$ for these objects are, respectively, $0.330 \pm 0.06,0.358 \pm 0.03,0.269 \pm 0.02$, $0.114 \pm 0.23$, and $0.245 \pm 0.09$.

Among these objects only HD 100764, a non-pulsating R-type carbon star, has previous polarimetric measurements in literature. Percentage polarization estimates in the $V$ band on two occasions by Parthasarathy et al. (2005) are, respectively, $0.20 \pm 0.05$ (JD 2,447,971) and 0.42 \pm 0.14 , (JD 2,448,398). Multiband photo-polarimetry of HD 100764 shows no significant variations in $p \%$ in $B, V, U$ and $I$ bands. No information is available on time variability of polarization for this star. Our estimated $p \%=0.33 \pm 0.06$ is not too different from the previous estimates.

The reddening in the direction of HD $100764(E(B-V)=0.02$; Eggen 1972) is consistent with the high Galactic latitude of the star. The observed $V$-band polarization is greater than the mean interstellar contribution to the polarization, indicating that the star is intrinsically polarized. Richer (1975) derived the absolute visual magnitude of $M_{v}=+0.3$ for this object that corresponds to a distance of $500 \mathrm{pc}$ with $E(B-V)=0.02$. From a detailed spectroscopic analysis Dominy (1984) found $T_{\text {eff }}=4850 \mathrm{~K}, \log g=2.2$ and a metallicity $[\mathrm{Fe} / \mathrm{H}]=-0.6$ for this object.

HD 100764 shows significant far-infrared excess fluxes at $12 \mu \mathrm{m}, 25 \mu \mathrm{m}, 60 \mu \mathrm{m}$ and $100 \mu \mathrm{m}$ with uncertainties of $4 \%, 8 \%, 12 \%$, and $12 \%$, respectively (Beichmann et al. (1985)). The silicate dust shell around HD 100764 is believed to absorb the radiation from the star and re-emit the radiation in the infrared. Modeling of its circumstellar dust by Skinner (1994) 
revealed a massive dusty disk. Parthasarathy (1991) suggested that HD 100764 is a star with a detached cold dust shell and that the optical region fluxes of HD 100764 do not show evidence for significant reddening, and argued that the dust shells are optically thin with relatively large dust grain size and dust is confined in the form of thin disc around the star and hence shows less reddening. The silicate dust shells around this star may be the result of mass loss experienced by the star during core helium flash, which has taken place recently. The presence of dust shell around HD 100764 also suggests that it has experienced significant mass loss in the recent past.

The star HD 168986 is listed in the Barium star catelog of Lü (1991). Barium stars are however not known to have dust shells or discs around them (Dominy et al. 1986). This star mentioned as a peculiar star in SIMBAD shows an estimated $p \%=0.36 \pm 0.03$, that seems to represent the intrinsic polarization property of the star. No previous polarization estimates are available for this object.

HD 198269 is listed in the CH star catalog of Bartkevicius (1996). In SIMBAD this star is mentioned as a star with envelop of CH. Lee (1974) gave an upper limit to its distance estimate as $\sim 750 \mathrm{pc}$ that corresponds to $M_{v}=-1.66$, an upper limit to the absolute magnitude. This is a non-variable $\mathrm{CH}$ star of type $\mathrm{R}$ with a mass of about 0.8 $M_{\odot}$ (Wallerstein 1973) and is similar in chemical compositions in comparison to other $\mathrm{CH}$ stars. With $E(B-V) \leq 0.07$ mag for this star, the estimated $p \%=0.27 \pm 0.29$ is marginally higher than the mean interstellar contribution to the polarization. No previous polarization estimates are available for this object.

HD 209621 and HE 1152-0355, both are confirmed CH stars. Surface composition of these two objects show abundance patterns that are consistent with the abundances generally noticed in $\mathrm{CH}$ stars, essentially arising from $s$-processing (Goswami \& Aoki (2010a); Goswami et al. (2006)). HD 209621 shows high abundances of $r$-process element Eu as well as of the third-peak $s$-process element $\mathrm{Pb}$. $\mathrm{Eu}$ and $\mathrm{Pb}$ are, however, not detected in the spectrum of HE 1152-0355. Both these objects show larger enhancement of the secondpeak $s$-process elements as compared to those of the first-peak $s$-process elements. Both are high-velocity objects. HD 209621 is a radial velocity variable with a period of 407.4 days (McClure \& Woodsworth 1990); however, radial velocity variability is not yet confirmed for HE 1152-0355. Our $V$-band polarimetric estimates indicate no significant polarization from HD 209621, and HE 1152-0355 shows polarization that is marginally above the mean interstellar contribution to the polarization. 


\subsection{HE 1027-2501, HE 1305+0007, HE 1429-0551, LP 625-44, HE 1523-1155}

These objects show significant polarization with $p \% \geq 1$, except for HE 1523-1155 for which we have obtained $p \%=0.849 \pm 0.08$. Reddening estimates $E(B-V)$ range from 0.03 to 0.08 for these stars; the values of $E(B-V)$ for the HE stars are taken from Beers et al. (2007).

A low carbon isotopic ratio $\left({ }^{12} \mathrm{C} /{ }^{13} \mathrm{C}<10\right)$ for $\mathrm{HE} 1027-2501$ indicates that the star is on the first ascent of the giant branch wherein the material transferred from the now unseen companion has been mixed into the CN-burning region of the $\mathrm{CH}$ star or constitute a minor fraction of the envelop mass of the $\mathrm{CH}$ star. Such low values are believed to be due to convection which dredges up the products of internal CNO cycle to the stellar atmosphere in the ascending red giant branch (RGB). When the star reaches the AGB stage, fresh ${ }^{12} \mathrm{C}$ may be supplied from the internal He-burning layer to the stellar surface, leading to an increase of ${ }^{12} \mathrm{C} /{ }^{13} \mathrm{C}$ ratio.

HE $1305+0007$ shows enhancement of both $r$ - and $s$-process elements including lead. The second-peak $s$-process elements are more enhanced than the first-peak $s$-process elements. This is a low-metallicity and high-velocity object $\left(V_{r}=+217.8 \pm 1.5 \mathrm{~km} \mathrm{~s}^{-1}\right)$; its atmospheric parameters are consistent with a present location on the RGB (Goswami et al. (2006)).

The star LP 625-44 is a carbon- and s-process-element-rich very metal-poor subgiant. Abundance estimate derived using the O I triplet around $7770 \AA$ shows excess of oxygen by a factor of 10 (Aoki et al. 2002); and Na enhancement by about a factor of 50 in comparison to HD 140283 (a metal-poor subgiant with normal abundance ratio) and a high Mg abundance $([\mathrm{Mg} / \mathrm{Fe}]=1.12 \pm 0.24)$. High abundance of Na suggests, that hydrogen burning in the ${ }^{22} \mathrm{Ne}$-rich layer in an AGB star must have produced the abundance pattern of this object. The $\mathrm{Pb}$ enhancement shown by LP 625-44 is not high enough to be placed in the group of lead stars. The abundance ratio of $s$-process elements at the second peak (La, Ce, and $\mathrm{Nd}$ ) to that at the third peak $(\mathrm{Pb})$ in LP 625-44 is significantly higher (by a factor of 5) than that in the $s$-process element-rich lead star HD 196944 (Van Eck et al. (2001)). Unlike CH stars, the radial velocity of this object is low $\left(\sim 30 \mathrm{~km} \mathrm{~s}^{-1}\right)$ and the variation of the radial velocity is expected to be for about 200 days (Aoki et al. 2000).

Like LP 625-44, HE 1429-0551, and HE 1523-1155 are also low-velocity objects $(\sim-44.9$ and $\sim-46.03 \mathrm{~km} \mathrm{~s}^{-1}$, respectively). Both show high abundances of $\mathrm{C}, \mathrm{N}$, and $\mathrm{Mg}$ relative to

Fe. Estimated carbon isotopic ratio of ${ }^{12} \mathrm{C} /{ }^{13} \mathrm{C}$ for $\mathrm{HE} 1429-0551$ is high $\sim 30_{-10}^{+20}$ (Aoki et al. 2007). Such high ratios are generally noticed in C-N stars. With a marginal difference in the 
molecular band depths, the spectra of the star HE 1523-1155 closely resemble the spectrum of HD 5223, a well-known CH giant (Goswami 2005). Both the HE stars show high barium abundance with respect to $\mathrm{Fe}([\mathrm{Ba} / \mathrm{Fe}]=1.57$ and 1.72 , respectively $)$, and $\log \left(L / L_{\odot}\right)$ are, respectively, 2.56 and 2.50 (Aoki et al. 2007). The observed $V$-band polarization estimates are markedly higher than the mean interstellar contribution to the polarization and seem to represent the stars intrinsic polarization properties.

\section{Conclusions}

Our $V$-band polarimetric observations presented for a sample of 10 carbon-enhanced metal-poor stars show five objects with polarization $p \%>1$ and the other five with $p \%<$ 0.4. While the size of the sample is relatively small, it should be noted that these stars belong to similar group in terms of carbon enhancement and metal deficiency. Thus, a clear separation of these objects into well-separated $V$-band polarimetric groups is significant. Intrinsic polarization of late-type stars are usually attributed either to the Rayleigh scattering of the light emerging from the limb of a star in which case the sources of photometric asymmetry necessary for a net observable polarization are suggested to be non-radial pulsation of the star, variation of temperature over the surface and the presence of giant convection cells (Harrington (1969); Schwarzschild (1975)) or due to scattering by molecules and dust grains in an extended asymmetric circumstellar envelope (Kruszewski et al. (1968); Shawl (1975); Daniel (1978)). It seems circumstellar grain scattering is the main mechanism responsible for the observed polarization in the sample of program stars. It would be worthwhile to conduct polarimetric observations for the entire sample of carbon stars from Hamburg ESO survey (Christlieb et al. 2001) for a statistical evaluation of their polarization properties.

Except for HD 100764, our results are the first estimates of polarization for the program stars. While the separation of the stars into two distinct polarization groups is a significant feature, it is not clear at the moment if this is a time-dependent property. While a few of the CEMP stars are confirmed as radial velocity variables and binaries, binarity of others are not yet established. Two important means for establishing variability and binarity of stars are long-term photometric observations as well as long-term spectroscopic radial velocity measurements. Time variation of polarization measurements for these objects would also be an indicator of their variability caused by the morphology of the circumstellar dust clouds that might have originated during AGB mass loss of the companion stars. Thus, the detection of polarization from these objects would lend support to those physical scenarios that consider binarity of the objects to explain their formation mechanism and peculiar abundances of heavy elements. 
Acknowledgement

We thank the staff at IUCCA observatory for time allocation and assistance during the observations. This work made use of the SIMBAD astronomical database, operated at CDS, Strasbourg, France, and the NASA ADS, USA. We thank Prof. A. V. Raveendran for useful discussions. Sreeja S. Kartha is a JRF in the DST project SR/S2/HEP-09/2007; funding from this project is greatfully acknowledged.

\section{REFERENCES}

Aoki, W., Norris, J. E., Ryan, S. G., Beers, T. C., \& Ando, H. 2000, ApJ, 536, L97.

Aoki, W., et al. 2002, PASJ, 54, 427

Aoki, W., et al. 2007, ApJ, 655, 492

Bartkevicius, A., 1996, Balt. Astron., 5, 217

Beers, T. C., et al. 2007, ApJS, 168, 128

Beichmann, C. A., Neugebauer, G., Habing, H. J., Clegg, P. E., \& Chester, T. J., 1985, IRAS Point Sources Catalog, (Pasadena, CA: JPL)

Chavero, C., Gomez, M., Whitney, B. A., \& Saffe, C. 2006, A\&A, 452, 921

Christlieb, N., Green, P. J., Wisotzki, L., \& Reimers, D. 2001, A\&A, 375, 366

Clarke, D., Smith, R. A., \& Yudin, R. V. 1998, A\&A, 336, 604

Czyzak, S. J., Hirth, J. P., \& Tabak, R. G. 1982, Vistas Astron., 25, 337

Daniel, J. Y. 1978, A\&A, 67, 345

Dominy, J. F. 1984, ApJS, 55, 27

Dominy, J. F., Lambert, D. L., Gehrz, K. D., \& Mozurkewich, D. 1986, AJ, 91, 591

Drake, N. A. \& Pereira, C. B. 2008, AJ, 135, 1070

Eggen, O. 1972, MNRAS, 159, 403

Goswami, A. 2005, MNRAS, 359, 531 
Goswami, A., \& Aoki, W. 2010a, MNRAS, 404, 253

Goswami, A., Aoki, W., Beers, T. C., Christlieb, N., Norris, J. E., Ryan, S. G., \& Tsangarides, S. 2006, MNRAS, 372, 343

Goswami, A., Bama, P., Shantikumar, N. S., \& Devassy, D. 2007, BASI, 35, 339

Goswami, A., Karinkuzhi, D., \& Shantikumar, N. S. 2010b, MNRAS, 402, 1111

Harrington, J. P., 1969, ApJ, 3, 165

Johnson, J. J. \& Jones, T. J. 1991, AJ, 101, 1735

Kruszewski, A., Gehrels, T., \& Serkowski, K. 1968, AJ, 73, 677

Lee, P. 1974, ApJ, 192, 133

Leonard, D. C., Li, W., Filippenko, V., Foley, R. J., \& Chornock, R., 2005, ApJ, 632, 450

Lü P. K. 1991, AJ, 101, 2229

McClure, R. D. \& Woodsworth, A. W. 1990, ApJ, 352, 709

McDavid, D. 1999, PASP, 111, 494

Parthasarathy, M., 1991, A\&A, 247, 429

Parthasarathy, M. \& Jain, S. K. 1993, in IAU Symp. 155, Planetary Nebulae, ed. R. Weinberger \& A. Acker ( Dordrecht: Kluwer), 353

Parthasarathy, M., Jain, S. K., \& Sarkar, G., 2005, AJ, 129, 2451

Ramprakash, A. N., Gupta, R., Sen, A. K., \& Tandon, S. N. 1998, A\&AS, 128, 369

Rautela, B. S., Joshi, G. C., \& Pandey, J. C. 2004, BASI, 32, 159

Raveendran, A. V. 1991, A\&A, 243, 453

Reiz, A. \& Franco, G. A. P. 1998, A\&AS, 130, 133

Richer, H. B. 1975, ApJ, 197, 611

Rowan-Robinson, M. \& Harris, S. 1983, MNRAS, 202, 797

Schwarzschild, M. 1975, ApJ, 195, 137

Sen, A. K., Gupta, R., Ramprakash, A. N.,Tandon, S. N. 2000, A\&AS, 141, 175 
Serkowski, K. 1974, in Planets, Stars, and Nebulae Studied with Photopolarimetry, ed. T. Gehrels (Tuscon, AZ: Univ. Arizona Press), 135

Serkowski, K., Mathewson, D. S., \& Ford, V. L. 1975, ApJ, 196, 261

Shawl, S. J. 1975, AJ, 80, 595

Skinner, C. J. 1994, MNRAS, 271, 300

Trammell, S. R., Dinerstein, H. L. \& Goodrich, R. W. 1994, AJ, 108, 984

Turnshek, D. A., Bohlin, R. C., Williamson, R. L., Lupie, O. L., Koornneef, J., \& Morgan, H. D. 1990, AJ, 99, 1243

Van Eck, S., Goriely, S., Jorissen, A., \& Plez, B. 2001, Nature, 412, 793

Wallerstein, G. 1973, ARA\&A, 11, 115

Weitenbeck, A. J. 2004, Acta Astron., 54, 87

This preprint was prepared with the AAS IATEX macros v5.2.

Table 1: Basic Parameters of the Program Stars

\begin{tabular}{|c|c|c|c|c|c|c|c|c|c|}
\hline Stars & R.A.(2000) & Decl.(2000) & $+l$ & $b$ & $V$ & $J$ & $H$ & $K_{s}$ & Date of Obs. \\
\hline HE $1027-2501$ & 102929.5 & $\begin{array}{lll}-25 & 17 & 16.2\end{array}$ & 266.6829 & +27.4161 & 12.70 & 10.627 & 9.896 & 9.722 & 2009 Apr 30 \\
\hline HD $94851^{*}$ & 105644.2 & -203952.6 & 269.8196 & +34.7281 & 9.27 & 8.869 & 8.816 & 8.771 & 2009 Apr 30 \\
\hline HD100764 & 113542.7 & -143536.6 & 276.8586 & +44.4107 & 8.73 & 7.048 & 6.60 & 6.513 & 2009 Apr 30 \\
\hline HE $1152-0355$ & 115506.1 & -041224.0 & 277.3246 & +55.8385 & 11.43 & 9.339 & 8.665 & 8.429 & 2009 Apr 30 \\
\hline HE $1305+0007$ & 130803.8 & $-0008 \quad 47.4$ & 311.9430 & +62.4340 & 12.22 & 10.247 & 9.753 & 9.600 & 2009 Apr 30 \\
\hline HE $1429-0551$ & 143231.3 & $\begin{array}{lll}-06 & 05 & 00.2\end{array}$ & 343.0186 & +48.7605 & 12.60 & 10.734 & 10.272 & 10.066 & 2009 Apr 30 \\
\hline HE $1523-1155$ & 152641.0 & -120542.6 & 351.8699 & +35.6301 & 13.22 & 11.372 & 10.846 & 10.748 & 2009 Apr 30 \\
\hline HD $147084^{*}$ & 162038.1 & $-24 \quad 1009.5$ & 352.3279 & +18.0503 & 4.55 & 2.222 & 1.899 & 1.681 & 2009 Apr 30 \\
\hline LP $625-44$ & 164314.0 & -015530.2 & 015.1463 & +27.1749 & 11.85 & 10.432 & 10.058 & 10.825 & 2009 Apr 30 \\
\hline HD $160529^{*}$ & 174159.0 & $\begin{array}{lll}-33 & 30 & 13.7\end{array}$ & 355.7021 & -01.7322 & 6.77 & 3.547 & 3.056 & 2.790 & 2009 Apr 30 \\
\hline HD 168986 & $1823 \quad 10.1$ & -153636.5 & 015.8506 & -00.9879 & 9.21 & 6.901 & 6.379 & 6.184 & 2009 Apr 30 \\
\hline HD 196944 & 204046.0 & -064750.6 & 039.5635 & -27.3703 & 8.41 & 7.017 & 6.626 & 7.504 & 2009 Apr 30 \\
\hline HD 198269 & $2048 \quad 36.7$ & +175023.7 & 063.2273 & -15.9516 & 8.12 & 6.078 & 5.505 & 5.385 & 2009 Apr 30 \\
\hline HD 206983 & 214609.3 & $-15 \quad 1439.7$ & 038.6462 & -45.4725 & 9.45 & 6.934 & 6.247 & 6.035 & 2009 Apr 28 \\
\hline HD209621 & 220425.1 & +210308.9 & 078.6453 & -27.1175 & 8.86 & 6.661 & 6.045 & 5.913 & 2009 Apr 28 \\
\hline
\end{tabular}

Note. The stars marked with $*$ are polarization standard stars. 

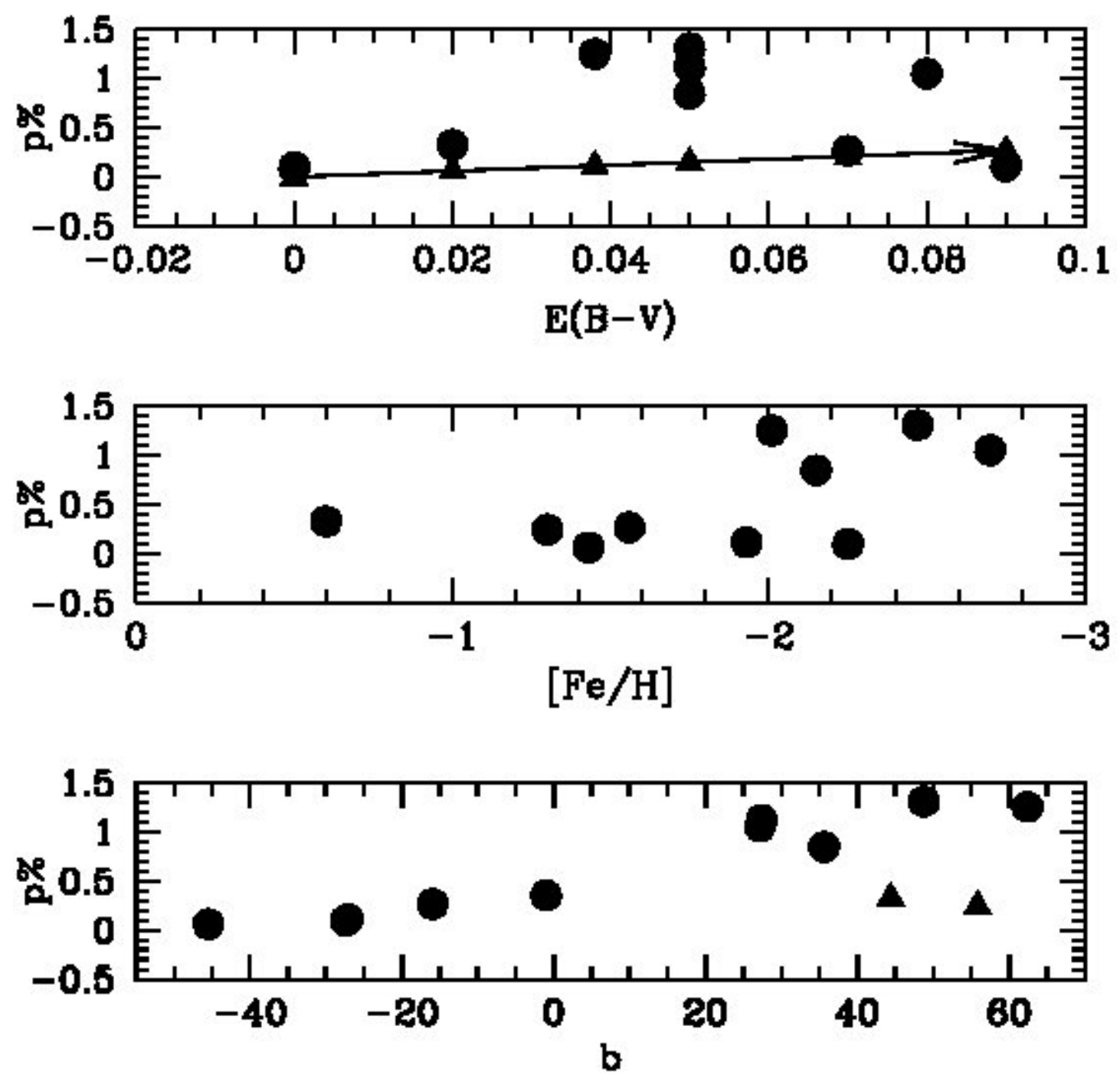

Fig. 1.- Upper panel: observed $V$-band percentage polarization for the program stars vs. interstellar $E(B-V)$. The solid line represents the mean interstellar polarization ( $3 E(B-V))$. Middle panel: observed $V$-band percentage polarization of the program stars w.r.t. their metallicities $[\mathrm{Fe} / \mathrm{H}]$. Polarization does not seem to have any co-relation w.r.t. metallicities of the stars. Bottom panel: the observed $V$-band percentage polarizations of the program stars are plotted w.r.t. their Galactic latitudes. Estimated polarizations are larger for objects with positive Galactic latitudes; however, there are two outliers (shown with triangles) that show weaker polarization. These two objects (HD 100764 and HE 11520355 with $[\mathrm{Fe} / \mathrm{H}] \sim-0.6$ and -1.3 , respectively) are not so metal-poor compared to the other stars that show larger polarization. 
Table 2: $V$-band Polarimetry of Polarization Standard Stars

\begin{tabular}{|c|c|c|c|c|c|c|}
\hline $\begin{array}{c}\text { Star Names } \\
(1)\end{array}$ & $\begin{array}{l}\text { HJD } \\
(2) \\
\end{array}$ & $\begin{array}{l}p \% \\
(3)^{*}\end{array}$ & $\begin{array}{c}\theta^{0} \\
(4)^{*}\end{array}$ & $\begin{array}{l}p \% \\
(5)\end{array}$ & $\begin{array}{l}\theta^{0} \\
(6)\end{array}$ & $\begin{array}{c}\text { Remarks } \\
(7)\end{array}$ \\
\hline HD 94851 & 2454952.13280 & $0.11 \pm 0.12$ & & $\begin{array}{c}0.057 \pm 0.018 \\
0.134 \\
\leq 0.1\end{array}$ & & 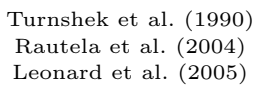 \\
\hline HD 147084 & 2454952.25726 & $4.12 \pm 0.02$ & 32.2 & $\begin{array}{c}4.16 \pm 0.01 \\
4.17 \pm 0.008 \\
4.19 \pm 0.03 \\
4.23 \\
4.21\end{array}$ & $\begin{array}{l}32.1 \pm 1.9 \\
32.9 \\
32.4 \pm 0.9 \\
33.5 \\
31.3\end{array}$ & 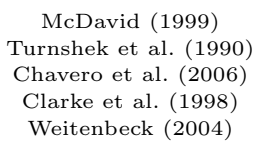 \\
\hline HD 160529 & 2454952.46513 & $7.45 \pm 0.02$ & 20.9 & $\begin{array}{c}7.35 \pm 0.55 \\
7.3 \pm 0.54 \\
7.52\end{array}$ & $\begin{array}{c}20 \\
20.1\end{array}$ & $\begin{array}{c}\text { Reiz \& Franco } 1998 \\
\text { Serkowski }(1974) \\
\text { Clarke et al. } \\
\text { Cla98 }\end{array}$ \\
\hline
\end{tabular}

Notes. Estimates listed in Columns 5 and 6 are from references listed in Column 7 .

* Our estimates of $p \%$ and offset corrected position angles (magnitudes)

Table 3: $V$-band Polarimetry of Carbon Stars

\begin{tabular}{lcccccccc}
\hline Star Names & $E(B-V)$ & $T_{\text {eff }}$ & $\log g$ & {$[\mathrm{Fe} / \mathrm{H}]$} & Reference & HJD & $\mathrm{p} \%$ & $\theta^{0}$ \\
\hline HD 209621 & 0.09 & 4500 & 2.0 & -1.93 & 1 & 2454950.47784 & $0.11 \pm 0.23$ \\
HD 206983 & - & 4200 & 1.4 & -1.43 & 2 & 2454950.48532 & $0.06 \pm 0.13$ \\
HD 100764 & 0.02 & 4850 & 2.2 & -0.6 & 3 & 2454952.14664 & $0.33 \pm 0.06$ & 4.0 \\
HE 1152-0355 & 0.026 & 4000 & 1.0 & -1.3 & 4 & 2454952.15514 & $0.24 \pm 0.09$ & 30.5 \\
HE 1027-2501 & 0.05 & - & - & - & - & 2454952.18021 & $1.13 \pm 0.11$ & 12.4 \\
HE 1305+0007 & 0.038 & 4750 & 2.0 & -2.01 & 4 & 2454952.22191 & $1.26 \pm 0.04$ & 41.9 \\
HE 1429-0551 & 0.05 & 4700 & 1.5 & -2.47 & 5 & 2454952.27449 & $1.31 \pm 0.06$ & 28.9 \\
LP 625-44 & 0.08 & 5500 & 2.5 & -2.70 & 6 & 2454952.31728 & $1.06 \pm 0.03$ & 8.0 \\
HE 1523-1155 & 0.05 & 4800 & 1.6 & -2.15 & 5 & 2454952.35635 & $0.85 \pm 0.08$ & 30.3 \\
HD 168986 & - & - & - & - & - & 2454952.39775 & $0.36 \pm 0.03$ & 32.5 \\
HD 196944 & 0.0 & 5250 & 1.8 & -2.25 & 7 & 2454952.47013 & $0.09 \pm 0.02$ & \\
HD 198269 & $<0.07$ & 4460 & - & -1.56 & 8 & 2454952.47835 & $0.27 \pm 0.02$ & 39.5
\end{tabular}

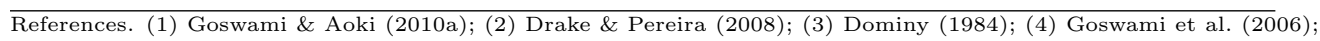

(5) Aoki et al. (2007); (6) Aoki et al. (2002); (7) Van Eck et al. (2001); (8) Lee (1974). 